\title{
Study on the relationship between the expression of S100A12, CaSR, and IL-7R in the synovium of knee osteoarthritis and angiogenesis
}

\author{
Min $\mathrm{Xu}^{1,2 \#}$, Jia Tu ${ }^{1,2 \#}$, Yanwei Yang ${ }^{1,2}$, Wenjuan Zou ${ }^{1,2}$ \\ ${ }^{1}$ Orthopedics of Traditional Chinese Medicine, Sichuan Provincial People's Hospital, University of Electronic Science and Technology of China, \\ Chengdu, China; ${ }^{2}$ Chinese Academy of Sciences, Sichuan Translational Medicine Research Hospital, Chengdu, China \\ Contributions: (I) Conception and design: M Xu, J Tu; (II) Administrative support: Y Yang, W Zou; (III) Provision of study materials or patients: \\ M Xu; (IV) Collection and assembly of data: J Tu, Y Yang; (V) Data analysis and interpretation: M Xu, J Tu; (VI) Manuscript writing: All authors; \\ (VII) Final approval of manuscript: All authors. \\ "These authors contributed equally to this work. \\ Correspondence to: Yanwei Yang; Wenjuan Zou. Orthopedics of Traditional Chinese Medicine, 32 West Section 2, First Ring Road, Qingyang District, \\ Chengdu 610072, China. Email: Xu610000m@163.com; Zouwenjuan61@163.com.
}

\begin{abstract}
Background: To analyze the relationship between the expression of calcium binding protein (S100A12), calcium sensitive receptor (CaSR), and interleukin-7 receptor (IL-7R) in the synovium of knee osteoarthritis (KOA) and angiogenesis.

Methods: The clinical data of 92 patients with KOA admitted to Sichuan Provincial People's Hospital from February 2019 to May 2020 were collected (KOA group, Kellgren-Lawrence staging: 23 cases of grade 2, 49 cases of grade 3 , and 20 cases of grade 4). In addition, 87 cases of patients with normal knee cartilage that were not involved during the same period of treatment in this hospital were selected. The expressions of S100A12, CaSR, IL-7R, and microvascular density (MVD) were compared among different populations, and the correlation between S100A12, CaSR, IL-7R, and MVD, and its diagnostic value for KOA were analyzed.

Results: The values of S100A12, CaSR, IL-7R, and MVD in the KOA group were significantly higher than those in the control group $(\mathrm{P}<0.05)$; the values of S100A12, CaSR, and IL-7R in grade 4 patients were significantly higher than those in grade 2 and 3 patients $(\mathrm{P}<0.05) ;$ S100A12, CaSR, IL-7R, and MVD were all positively correlated, and the difference was statistically significant $(\mathrm{P}<0.05)$. According to the receiver operating characteristic (ROC) curve, the area under the curve (AUC) of S100A12, CaSR, and IL-7R for KOA diagnosis was 0.982, 0.929, and 0.899, respectively. The Youden index was 0.630, 0.835, 0.739, respectively.
\end{abstract}

Conclusions: The expression levels of S100A12, CaSR, and IL-7R in the synovium of KOA are significantly up-regulated, and they are closely related to angiogenesis.

Keywords: S100A12; CaSR; IL-7R; knee osteoarthritis (KOA); angiogenesis

Submitted Nov 11, 2021. Accepted for publication Dec 16, 2021.

doi: 10.21037/apm-21-3506

View this article at: https://dx.doi.org/10.21037/apm-21-3506

\section{Introduction}

Knee osteoarthritis (KOA) is a common clinical joint disease, the incidence of which increases with age, making it the main cause of joint pain and disability in the elderly (1). At present, the main treatment for KOA is to relieve pain, and prevent and delay cartilage degradation. However, long-term conservative treatment and expensive surgical treatment have brought tremendous psychological pressure and economic burden to patients. Some studies have found that gene therapy for osteoarthritis can enable target cells 
to obtain new biological behaviors and functions, and antagonize pathogenic factors by long-term expression of gene protein products in target cells, so as to achieve the purpose of preventing and treating $\mathrm{KOA}(2)$.

Calcium binding protein (S100A12) is a member of the $\mathrm{S} 100$ protein family. It can bind to a variety of ions, participate in the process of immune regulation and signal transduction, and regulate the activity of inflammatory cells. The expression of S100A12 is significantly upregulated in rheumatoid arthritis, tumor, and ulcerative colitis (3). The imbalance of extracellular matrix synthesis and degradation of articular cartilage in patients is one of the important reasons for cartilage degeneration, and the activation of calcium sensitive receptor (CaSR) can promote bone reconstruction, which is related to osteoarthritis (4). Interleukin-7 receptor (IL-7R) is expressed in B progenitor cells, T cells, lymphatic precursors, and other cells. It has been found that the expression of IL-7R is significantly increased on the surface of immune cells in patients with rheumatoid arthritis, and the severity of knee joint damage in patients with rheumatoid arthritis is reduced after blocking the expression of IL-7R (5). Other studies have confirmed that angiogenesis exists in OA synovium and osteochondral tissues. The markers of angiogenesis in synovium are correlated with the histological changes of synovitis, suggesting that angiogenesis is involved in the formation of chronic synovitis, and angiogenesis is closely related to the activation of endothelial cells and protease degradation by inflammatory mediators (6). At present, there are few studies on the production of S100A12, CaSR and IL-7R in synovial blood perfusion. Based on this, this study makes a preliminary exploration to analyze the expression of S100A12, CaSR and IL-7R in the synovial membrane of KOA and its relationship with angiogenesis, aiming to provide a theoretical basis for the clinical treatment of KOA. We present the following article in accordance with the STARD reporting checklist (available at https://dx.doi.org/10.21037/apm-21-3506).

\section{Methods}

\section{General information}

The clinical data of 92 patients with KOA (KOA group) admitted to Sichuan Provincial People's Hospital from February 2019 to May 2020 were collected, including 51 males and 41 females, aged 54-73 years, with an average age of $65.24 \pm 3.41$ years; Kellgren-Lawrence $(\mathrm{KL})$ staging:
23 cases of grade 2, 49 cases of grade 3, and 20 cases of grade 4 . The inclusion criteria were as follows: (I) articular fluid examination in line with the standard clear and viscous, pericytes count $<2 \times 10^{9} / \mathrm{L}$; (II) knew and agreed to participate in this study, with the provision of written informed consent; (III) meeting the diagnostic criteria for KOA revised by the American Rheumatism Association (6); (IV) X-ray examination showed that the joint space had narrowed and osteophytes appeared at the knee joint; (V) bone friction sounds during activity. The exclusion criteria were as follows: (I) patients with severe primary diseases such as those of the heart, liver, and kidney; (II) history of joint replacement surgery; (III) having received intraarticular injection within half a year; (IV) treatment with glucocorticoids within 2 weeks. In addition, 87 patients who did not have knee cartilage involvement in the same period of treatment in our hospital were selected, including 53 males and 34 females, aged 54-77 years, with an average of $66.30 \pm 4.00$ years. Among them, there were 56 cases of tibial fracture and supracondylar fracture of the femur were fixed with intramedullary nail, and 31 cases of meniscus and ligament injury. There was no difference in the general data of age and gender among the participants $(\mathrm{P}>0.05)$, which was comparable. All procedures performed in this study involving human participants were in accordance with the Declaration of Helsinki (as revised in 2013). The study was approved by ethics committee of Sichuan Provincial People's Hospital [Lunshen (Research) No. 26, 2016] and informed consent was taken from all the patients.

\section{Study methods}

\section{Detection methods of S100A12, CaSR, and IL-7R}

We extracted synovial tissue from both participant groups before surgery. To ensure that knee suprapatellar bursa synovial tissue was aseptically maintained in vitro, it was placed directly in a sterile tube containing liquid nitrogen, and $30 \mathrm{~min}$ later was placed in a $-80{ }^{\circ} \mathrm{C}$ refrigerator for standby. Enzyme-linked immunosorbent assay (ELISA) was used to detect S100A12, and the kit was provided by Abnova (Walnut, CA, USA). The expression levels of CaSR and IL-7R were detected by real time polymerase chain reaction (RT-PCR), Total RNA was extracted by Trizol method, and the first strand of complementary DNA (cDNA) was synthesized by reverse transcription using an Applied Biosystems reverse transcription kit (Invitrogen, Waltham, MA, 
Table 1 Comparison of the expression of S100A12, CaSR, IL-7R and angiogenesis factors in different populations $(\bar{x} \pm \mathrm{s})$

\begin{tabular}{lccccc}
\hline Group & Case number & S100A12 $(\mathrm{ng} / \mathrm{mg})$ & CaSR & IL-7R & MVD \\
\hline Control group & 87 & $15.98 \pm 5.47$ & $1.17 \pm 0.57$ & $0.35 \pm 0.13$ & $13.07 \pm 2.40$ \\
KOA group & 92 & $27.71 \pm 7.90$ & $3.76 \pm 2.16$ & 11.107 & $10.351 \pm 0.78$ \\
$\mathrm{t}$ & - & 11.468 & $<0.001$ & $<0.001$ & 14.083 \\
$\mathrm{P}$ value & - & $<0.001$ & $<0.001$ & \\
\hline
\end{tabular}

MVD, microvascular density; KOA, knee osteoarthritis; S100A12, calcium binding protein S100A12; CaSR, calcium sensitive receptor; IL-7R, interleukin-7 receptor.

USA), and was operated according to the manufacturer's instructions. IL-7R forward primer sequence: 5'CTGTTGGTCATCTTGGCCTGT -3', reverse primer sequence: 5'- GCTTGAATGTCATCCACCCTAT -3'; CaSR forward primer sequence: 5'- TTCGGCATCAGCT TTGTG -3'; reverse primer sequence: 5'- TGAAGATGAT TTCGTCTTCC - 3 '.

\section{Detection of microvascular density}

Microvascular density (MVD) was defined as a single endothelial cell or a cluster of endothelial cells which stained positive for CD34 as a microvessel. For each stained section, we first selected 3 'points' under a low magnification, that is, the area with the highest MVD, and then counted the blood vessels at high magnification. The marginal reaction area was excluded. The obtained specimens were counted under 5 fields of view, and the average value was taken as the MVD value in this group.

\section{Observation indicators}

(I) Comparison of the expression of S100A12, CaSR, IL-7R, and angiogenic factors in different populations;

(II) Comparison of the expression of S100A12, CaSR, IL-7R, and angiogenic factors in different severities of disease;

(III) Correlation analysis of S100A12, CaSR, IL-7R, and angiogenic factors;

(IV) Analysis of the diagnostic value of S100A12, CaSR, and IL-7R in KOA.

\section{Statistical analysis}

The software SPSS 20.0 (IBM Corp., Chicago, IL, USA) was used to establish the database. Measurement data were described by mean \pm standard deviation $(\bar{x} \pm s)$, t/F test, and variance test was used between multiple groups.
The receiver operating characteristic (ROC) curve was drawn to analyze the diagnostic value of S100A12, CaSR, and IL-7R for KOA, and the area under the curve (AUC) was calculated. Pearson's test was used to analyze the correlation. $\mathrm{P}$ value $<0.05$ indicated that the difference was statistically significant.

\section{Results}

Comparison of the expression of S100A12, CaSR, $I L-7 R$, and angiogenic factors in different populations

The values of S100A12, CaSR, IL-7R, and MVD in the KOA group were significantly higher than those in the control group $(\mathrm{P}<0.05)$ (Table 1).

\section{Expression of S100A12, CaSR, IL-7R, and angiogenic factors in patients with different degrees of disease}

The values of S100A12, CaSR, and IL-7R in participants with grade 4 were significantly higher than those in participants with grade 2 and grade 3 , and the differences among the 3 groups were statistically significant $(\mathrm{P}<0.05)$, as shown in Table 2.

\section{Correlation of S100A12, CaSR, IL-7R and angiogenic factors}

A positive correlation was detected between S100A12, CaSR, IL-7R, and MVD, and the difference was statistically significant $(\mathrm{P}<0.05)$ (Table 3 and Figure 1$)$.

\section{Diagnostic value of S100A12, CaSR, and IL-7R in KOA}

According to the ROC curve, the AUC of S100A12, CaSR, and IL-7R in the diagnosis of KOAwere 0.982, 0.929, and 0.899 , respectively. The sensitivity and specificity were 
Table 2 Comparison of the expression of S100A12, CaSR, IL-7R, and angiogenesis factors in different populations $(\bar{x} \pm \mathrm{S})$

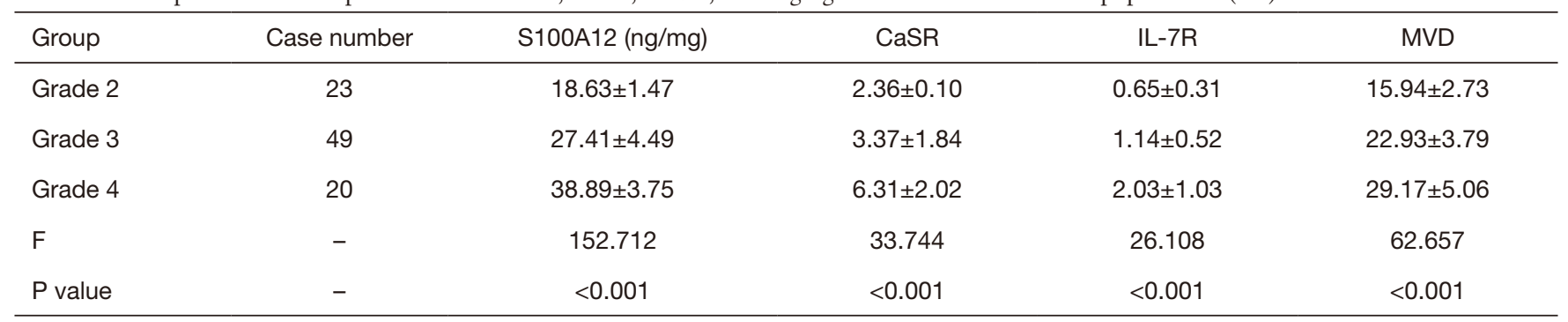

MVD, microvascular density; S100A12, calcium binding protein S100A12; CaSR, calcium sensitive receptor; IL-7R, interleukin-7 receptor.

Table 3 Correlation analysis of S100A12, CaSR, IL-7R, and angiogenesis factors

\begin{tabular}{lcc}
\hline Factor & $r$ & $P$ value \\
\hline S100A12 & 0.731 & $<0.01$ \\
CaSR & 0.672 & $<0.01$ \\
IL-7R & 0.696 & $<0.01$ \\
\hline
\end{tabular}

S100A12, calcium binding protein S100A12; CaSR, calcium sensitive receptor; IL-7R, interleukin-7 receptor.

(0.688, 0.942), $(0.882,0.953)$, and $(0.774,0.965)$, and the Youden index was $0.630,0.835,0.739$, respectively (Table 4 and Figure 2).

\section{Discussion}

The incidence of $\mathrm{KOA}$ is increasing annually, having a serious impact on people's daily life and work. The main pathological changes of KOA are due to the various progressive changes of the extracellular matrix, which affects the normal biomechanical characteristics of cartilage. However, the etiology of KOA is complex and still controversial. Studies have shown that synovitis plays an important role in the pathogenesis of KOA. Synovial tissue can secrete various active factors, which can stimulate bone resorption and osteoid cell proliferation, resulting in hyperplasia of subchondral bone and articular bone (7).

The expression of S100A12 happens mainly in neutrophils, which can be involved in tumor, inflammation, and metabolic diseases, and has a high affinity with calcium ions. After S100A12 binds to calcium ions or some inflammatory mediators, its protein conformation changes, thus exposing the binding site of the target protein, and binding with the corresponding ligand to play its biological function (8). Studies have shown that the expression of
S100A12 can hydrolyze extracellular matrix protein and enhance autoimmunity, which may be involved in the activity of osteoarthritis and the destruction of articular cartilage (9). Some studies have also shown that the proinflammatory effect of S100A12 in arthritis may be related to its high expression outside inflammatory arthritis cells, cell recruitment, and cytotoxicity. In particular, S100A12 can activate mast cells and participate in neutrophil metastasis (10).

A member of the $\mathrm{C}$ family in the $\mathrm{G}$ protein-coupled receptor, CaSR is composed of amino extracellular domain, 7 transmembrane helixes and transmembrane domains, and an intracellular carboxyl tail. Physically, CaSR is mainly involved in the regulation of calcium homeostasis, which is distributed in parathyroid, bone, kidney, and other tissues, as well as blood cells, peripheral nerve cells, and other cells. Its function is to maintain the homeostasis of $\mathrm{Ca}^{2+}$ and other metal ions, and also regulate cell differentiation, proliferation, and ion channel opening (11). Previous animal experiments showed that CaSR expression was found in cartilage tissue of a KOA animal model, and the expression of CaSR activity in osteogenesis and chondrocytes can promote bone reconstruction (12). Some studies have also shown that CaSR can promote the proliferation, differentiation, and mineralization of osteoblasts, indicating that apoptosis can be induced in osteoclasts (13).

The cytokine IL-7R is a heterodimer composed of IL-7 receptor $\alpha$ chain (IL-7R $\alpha$ ) and common $\gamma$ chain receptors. Previous studies have shown that IL-7R expression is significantly increased in synovial tissues of patients with rheumatoid arthritis, and its up-regulation is closely related to the expression of interleukin 7 (14). Some studies have found that the signaling pathway of IL-7R has a certain effect on chondrocytes. Chondrocytes can secrete IL-7, express IL-7R, and accept the stimulation signal transmitted by IL-7R, thereby secreting matrix metalloproteinase 13 , 

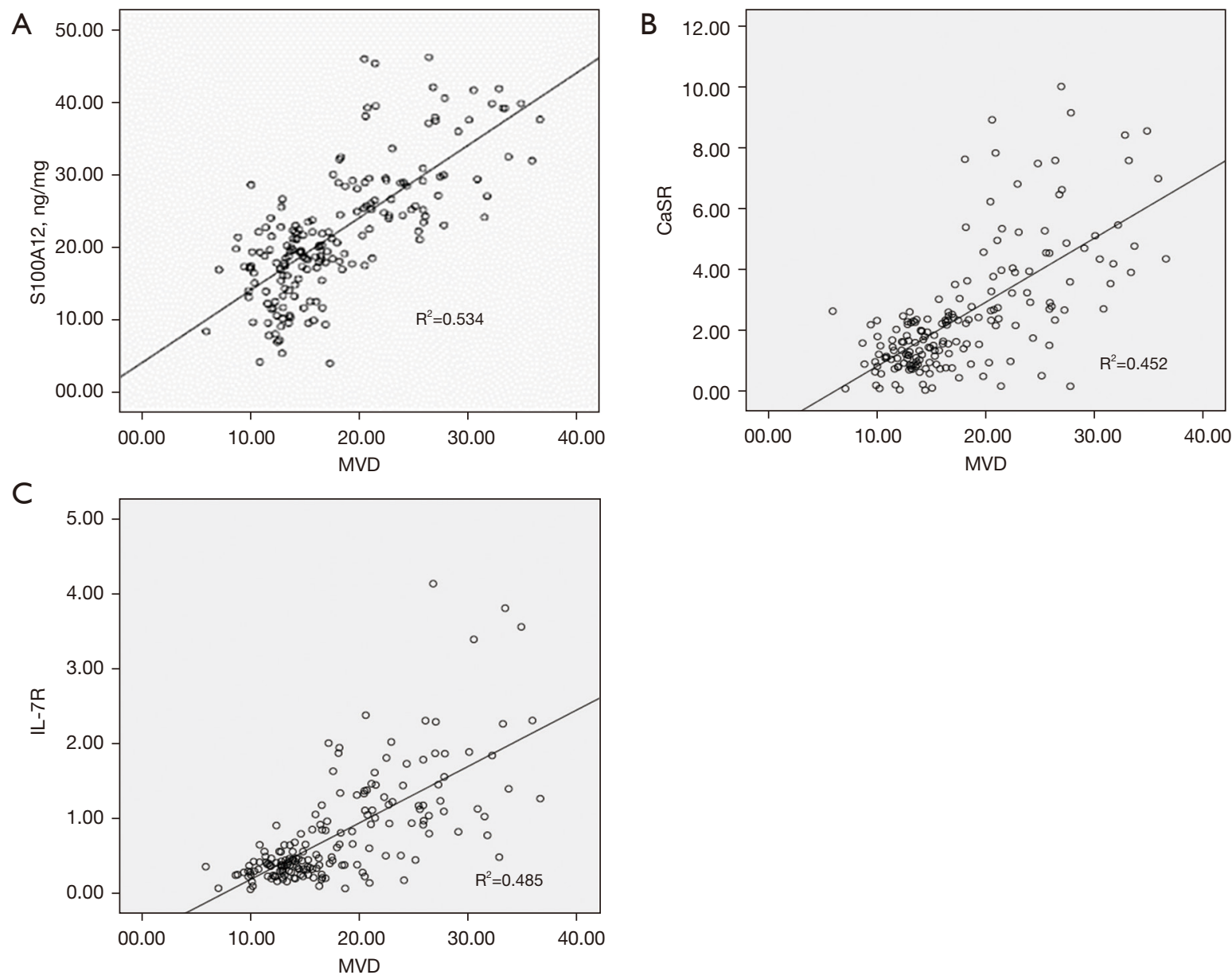

Figure 1 Correlation scatter plot of S100A12, CaSR, IL-7R, and MVD. MVD, microvascular density; S100A12, calcium binding protein S100A12; CaSR, calcium sensitive receptor; IL-7R, interleukin-7 receptor.

Table 4 Diagnostic value of S100A12, CaSR, and IL-7R in knee osteoarthritis

\begin{tabular}{|c|c|c|c|c|c|c|c|}
\hline \multirow{2}{*}{$\begin{array}{l}\text { Test result } \\
\text { variables }\end{array}$} & \multirow{2}{*}{$A \cup C$} & \multirow{2}{*}{$\begin{array}{c}\text { Progressive } \\
\text { Sig. value }\end{array}$} & \multicolumn{2}{|c|}{ Progressive $95 \% \mathrm{Cl}$} & \multirow{2}{*}{ Sensitivity } & \multirow{2}{*}{ Specificity } & \multirow{2}{*}{ Youden index } \\
\hline & & & Lower limit & Upper limit & & & \\
\hline S100A12 & 0.982 & 0.000 & 0.968 & 0.995 & 0.688 & 0.942 & 0.630 \\
\hline CaSR & 0.929 & 0.000 & 0.885 & 0.973 & 0.882 & 0.953 & 0.835 \\
\hline IL-7R & 0.899 & 0.000 & 0.846 & 0.951 & 0.774 & 0.965 & 0.739 \\
\hline
\end{tabular}

S100A12, calcium binding protein S100A12; CaSR, calcium sensitive receptor; IL-7R, interleukin-7 receptor; AUC, area under the curve; Youden index, the sum of sensitivity and specificity minus 1.

which has a degradation effect on the proteoglycan in cartilage (15). Therefore, it is proposed that IL-7R can be blocked to prevent cartilage degradation and alleviate bone damage.

The results of this study found that the expression of
S100A12, CaSR, and IL-7R in synovial tissue of participants with $\mathrm{KOA}$ was significantly higher than that of the control group, and the expression of S100A12, CaSR, and IL-7R increased significantly with the progression of the disease. Previous studies have shown that the expression of these 


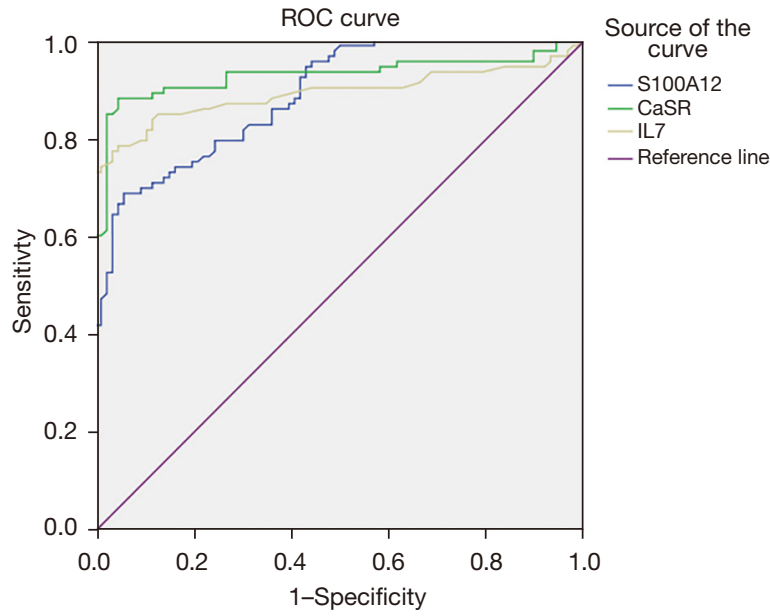

Figure 2 ROC curve of S100A12, CaSR and IL-7R in the diagnosis of KOA. ROC, receiver operating characteristic; KOA, knee osteoarthritis; S100A12, calcium binding protein S100A12; CaSR, calcium sensitive receptor; IL-7R, interleukin-7 receptor.

3 factors in the blood of patients with KOA was also significantly increased (16), which suggesting that S100A12, CaSR, and IL-7R may play an important role in the formation of knee synovial angiogenesis, and may also be an important cause of chronic and persistent knee synovitis. Angiogenesis refers to the formation of a new capillarybased vascular system based on the formed vascular system by bifurcation and germination. The MVD can reflect angiogenesis and has important application value in tumor prognosis evaluation (17). Increased neovascularization in synovial membrane of patients with KOA can cause adverse effects such as bone and cartilage destruction, and the formation of osteophytes (18). Previous studies have shown that there is a close relationship between angiogenesis and chronic inflammation in the progression of arthritis (19). In this study, it was found that S100A12, CaSR, and IL-7R were positively correlated with $\mathrm{MDV}$, and the increase of S100A12, CaSR, and IL-7R in patients with KOA may promote angiogenesis and further lead to the progression of the disease (20). The ROC curve showed that S100A12, CaSR, and IL-7R had certain value in the diagnosis and evaluation of KOA, which could provide a certain reference for clinical diagnosis and evaluation.

In summary, the expression of S100A12, CaSR, and IL-7R in synovial membrane of KOA was significantly upregulated, and was closely related to angiogenesis.

\section{Acknowledgments}

Funding: None.

\section{Footnote}

Reporting Checklist: The authors have completed the STARD reporting checklist. Available at https://dx.doi. org/10.21037/apm-21-3506

Data Sharing Statement: Available at https://dx.doi. org/10.21037/apm-21-3506

Conflicts of Interest: All authors have completed the ICMJE uniform disclosure form (available at https://dx.doi. org/10.21037/apm-21-3506). The authors have no conflicts of interest to declare.

Ethical Statement: The authors are accountable for all aspects of the work in ensuring that questions related to the accuracy or integrity of any part of the work are appropriately investigated and resolved. All procedures performed in this study involving human participants were in accordance with the Declaration of Helsinki (as revised in 2013). The study was approved by ethics committee of Sichuan Provincial People's Hospital [Lunshen (Research) No. 26, 2016] and informed consent was taken from all the patients.

Open Access Statement: This is an Open Access article distributed in accordance with the Creative Commons Attribution-NonCommercial-NoDerivs 4.0 International License (CC BY-NC-ND 4.0), which permits the noncommercial replication and distribution of the article with the strict proviso that no changes or edits are made and the original work is properly cited (including links to both the formal publication through the relevant DOI and the license). See: https://creativecommons.org/licenses/by-nc-nd/4.0/.

\section{References}

1. Xu X, Wan Y, Gong L, et al. Chinese herbal medicine Yanghe decoction for knee osteoarthritis: A protocol for systematic review and meta-analysis. Medicine (Baltimore) 2020;99:e21877.

2. Wei B, Zhang Y, Tang L, et al. Protective effects of 
quercetin against inflammation and oxidative stress in a rabbit model of knee osteoarthritis. Drug Dev Res 2019;80:360-7.

3. Wang Q, Aleshintsev A, Jose A, et al. Ca(II) and Zn(II) synergistically modulate the structure and selfssembly of Calgranulin C (S100A12). FASEB J 2020;34:1.

4. Sim JH, Kim JH, Park AK, et al. IL-7Ralow CD8+ T Cells from Healthy Individuals Are Anergic with Defective Glycolysis. J Immunol 2020;205:2968-78.

5. Goh SL, Persson MSM, Stocks J, et al. Relative Efficacy of Different Exercises for Pain, Function, Performance and Quality of Life in Knee and Hip Osteoarthritis: Systematic Review and Network Meta-Analysis. Sports Med 2019;49:743-61.

6. Henrotin Y, Donneau AF, de Vlam K, et al. Responses to "Bio-optimized Curcuma longa extract is efficient on knee osteoarthritis pain: a double-blind multicenter randomized placebo controlled three-arm study": authors' reply. Arthritis Res Ther 2020;22:23.

7. Veronese N. Reply to: "Bio-optimized Curcuma longa extract is efficient on knee osteoarthritis pain: a doubleblind multicenter randomized placebo controlled threearm study". Arthritis Res Ther 2020;22:21.

8. Wang XG, Gao LX, Zhao MW, et al. Relationship between quantitative magnetic resonance imaging and clinical symptoms in patients with knee osteoarthritis. Chin Med J (Engl) 2020;133:1741-3.

9. Weinhage T, Kölsche T, Rieger-Fackeldey E, et al. Cord Blood Low-Density Granulocytes Correspond to an Immature Granulocytic Subset with Low Expression of S100A12. J Immunol 2020;205:56-66.

10. Borsky P, Fiala Z, Andrys C, et al. Alarmins HMGB1, IL33, S100A7, and S100A12 in Psoriasis Vulgaris. Mediators Inflamm 2020;2020:8465083.

11. Zhang X, Ong C, Su G, et al. Characterization and engineering of S100A12-heparan sulfate interactions. Glycobiology 2020;30:463-73.

12. Ma JX, Wang B, Ding CF, et al. Couplet medicines of

Cite this article as: $\mathrm{Xu} M$, Tu J, Yang Y, Zou W. Study on the relationship between the expression of S100A12, CaSR, and IL-7R in the synovium of knee osteoarthritis and angiogenesis. Ann Palliat Med 2021;10(12):12775-12781. doi: 10.21037/apm-213506 leech and centipede granules improve erectile dysfunction via inactivation of the CaSR/PLC/PKC signaling in streptozotocin-induced diabetic rats. Biosci Rep 2020;40:BSR20193845.

13. Mayavan A, Krishnan SD, Rajendran P, et al. Silica nanoparticles assisted preparation of reddish-yellow emitting Eu2 + activated remote-type $\mathrm{CaSrSiO} 4$ phosphor for warm white LED applications. Ceramics International 2020;46:160-2.

14. Gao S, Chen Y, Zhao J, et al. Oat $\beta$-glucan ameliorates epidermal barrier disruption by upregulating the expression of CaSR through dectin-1-mediated ERK and p38 signaling pathways. Int J Biol Macromol 2021;185:876-89.

15. Veldeman L, Robbrecht S, Breckpot J, et al. A Case of a Heterozygous Inactivating CASR Variant with AdultOnset Symptomatic Hypercalcemia Requiring Extensive Surgery. Calcif Tissue Int 2020;107:104-8.

16. Singh P, Bhadada SK, Dahiya D, et al. Reduced Calcium Sensing Receptor (CaSR) Expression Is Epigenetically Deregulated in Parathyroid Adenomas. J Clin Endocrinol Metab 2020;105:dgaa419.

17. González-García S, Mosquera M, Fuentes P, et al. IL-7R is essential for leukemia-initiating cell activity of T-cell acute lymphoblastic leukemia. Blood 2019;134:2171-82.

18. Wang X, Peng H, Cong J, et al. Author Correction: Memory formation and long-term maintenance of IL$7 \mathrm{R} \alpha+\mathrm{ILC} 1 \mathrm{~s}$ via a lymph node-liver axis. Nat Commun 2019;10:160.

19. Leung GA, Cool T, Valencia CH, et al. The lymphoidassociated interleukin 7 receptor (IL7R) regulates tissue-resident macrophage development. Development 2019;146:dev176180.

20. Zhang MM, Beno BR, Huang RY, et al. An Integrated Approach for Determining a Protein-Protein Binding Interface in Solution and an Evaluation of HydrogenDeuterium Exchange Kinetics for Adjudicating Candidate Docking Models. Anal Chem 2019;91:15709-17. 